\title{
Approximating common fixed points of semigroups in metric spaces
}

\author{
Buthinah A Bin Dehaish ${ }^{1 *}$ and Mohamed A Khamsi2,3
}

\author{
"Correspondence: \\ bbindehaish@yahoo.com \\ ${ }^{1}$ Department of Mathematics, \\ Faculty of Science For Girls, King \\ Abdulaziz University, P.O. Box 80203, \\ Jeddah, 21589, Saudi Arabia \\ Full list of author information is \\ available at the end of the article
}

\begin{abstract}
In this paper, we investigate the common fixed points set of nonexpansive semigroups of nonlinear mappings $\left\{T_{t}\right\}_{t \geq 0}$, i.e., a family such that $T_{0}(x)=x$, $T_{s+t}=T_{s}\left(T_{t}(x)\right)$, where the domain is a metric space $(M, d)$. In particular we prove that under suitable conditions, the common fixed points set is the same as the common fixed points set of two mappings from the family. Then we use the modified Mann iteration process to approximate such common fixed points.

MSC: Primary 47H09; secondary 46B20; 47H10; 47E10

Keywords: fixed point; hyperbolic metric space; inequality; nearest point projection; Mann process; nonexpansive mapping; semigroup; uniformly convex metric space; uniformly Lipschitzian mapping
\end{abstract}

\section{Introduction}

The purpose of this paper is to prove the existence of common fixed points for semigroups of nonlinear mappings acting in metric spaces. Recently, Khamsi and Kozlowski presented a series of fixed point results for pointwise contractions, asymptotic pointwise contractions, pointwise nonexpansive and asymptotic pointwise nonexpansive mappings acting in modular function spaces $[1,2]$.

Let us recall that a family $\left\{T_{t}\right\}_{t \geq 0}$ of mappings forms a semigroup if $T_{0}(x)=x$ and $T_{s+t}=$ $T_{s} \circ T_{t}$. Such a situation is quite typical in mathematics and applications. For instance, in the theory of dynamical systems, the vector function space would define the state space and the mapping $(t, x) \rightarrow T_{t}(x)$ would represent the evolution function of a dynamical system. The question about the existence of common fixed points, and about the structure of the set of common fixed points, can be interpreted as a question whether there exist points that are fixed during the state space transformation $T_{t}$ at any given point of time $t$, and if yes - what the structure of a set of such points may look like. In the setting of this paper, the state space is a nonlinear metric space.

The existence of common fixed points for families of contractions and nonexpansive mappings in Banach spaces has been the subject of intense research since the early 1960s, as investigated by Belluce and Kirk [3, 4], Browder [5], Bruck [6], DeMarr [7], and Lim [8]. The asymptotic approach for finding common fixed points of semigroups of Lipschitzian (but not pointwise Lipschitzian) mappings has also been investigated, see, e.g., Tan and Xu [9]. It is worthwhile mentioning the recent studies on the special case, when the parameter set for the semigroup is equal to $\{0,1,2,3, \ldots\}$, and $T_{n}=T^{n}$, the $n$th iterate of an asymptotic

\section{Springer}

(c) 2015 Bin Dehaish and Khamsi; licensee Springer. This is an Open Access article distributed under the terms of the Creative Commons Attribution License (http://creativecommons.org/licenses/by/4.0), which permits unrestricted use, distribution, and reproduction in any medium, provided the original work is properly credited. 
pointwise nonexpansive mapping. Kirk and $\mathrm{Xu}$ [10] proved the existence of fixed points for asymptotic pointwise contractions and asymptotic pointwise nonexpansive mappings in Banach spaces, while Hussain and Khamsi [11] extended this result to metric spaces, and Khamsi and Kozlowski to modular function spaces [1, 2]. In the context of modular function spaces, Khamsi discussed in [12] the existence of nonlinear semigroups in Musielak-Orlicz spaces and considered some applications to differential equations.

\section{Uniform convexity in metric spaces}

Throughout this paper, $(M, d)$ will stand for a metric space. Suppose that there exists a family $\mathcal{F}$ of metric segments such that any two points $x, y$ in $M$ are endpoints of a unique metric segment $[x, y] \in \mathcal{F}([x, y]$ is an isometric image of the real line interval $[0, d(x, y)])$. We shall denote by $(1-\beta) x \oplus \beta y$ the unique point $\mathrm{z}$ of $[x, y]$ which satisfies

$$
d(x, z)=\beta d(x, y) \quad \text { and } \quad d(z, y)=(1-\beta) d(x, y),
$$

where $\beta \in[0,1]$. Such metric spaces are usually called convex metric spaces [13]. Moreover, if we have

$$
d\left(\frac{1}{2} p \oplus \frac{1}{2} x, \frac{1}{2} p \oplus \frac{1}{2} y\right) \leq \frac{1}{2} d(x, y)
$$

for all $p, x, y$ in $M$, then $M$ is said to be a hyperbolic metric space (see [14]).

Obviously, normed linear spaces are hyperbolic spaces. As nonlinear examples, one can consider the Hadamard manifolds [15], the Hilbert open unit ball equipped with the hyperbolic metric [16], and the CAT(0) spaces [17-19] (see Example 2.1). We will say that a subset $C$ of a hyperbolic metric space $M$ is convex if $[x, y] \subset C$ whenever $x, y$ are in $C$.

Definition 2.1 Let $(M, d)$ be a hyperbolic metric space. We say that $M$ is uniformly convex (in short, UC) if for any $a \in M$, for every $r>0$, and for each $\epsilon>0$,

$$
\delta(r, \varepsilon)=\inf \left\{1-\frac{1}{r} d\left(\frac{1}{2} x \oplus \frac{1}{2} y, a\right) ; d(x, a) \leq r, d(y, a) \leq r, d(x, y) \geq r \varepsilon\right\}>0 .
$$

The definition of uniform convexity finds its origin in Banach spaces [20]. To the best of our knowledge, the first attempt to generalize this concept to metric spaces was made in [21]. The reader may also consult [14, 16, 22].

From now onwards we assume that $M$ is a hyperbolic metric space and if $(M, d)$ is uniformly convex, then for every $s \geq 0, \epsilon>0$, there exists $\eta(s, \epsilon)>0$ depending on $s$ and $\epsilon$ such that

$$
\delta(r, \varepsilon)>\eta(s, \epsilon)>0 \quad \text { for any } r>s .
$$

Most of the results in this section may be found in [22].

Recall that the hyperbolic metric space $(M, d)$ is said to be strictly convex if whenever

$$
d(a, x)=d(a, y)=d(a, \lambda x \oplus(1-\lambda) y)
$$

for any $a, x, y \in M$ and $\lambda \in(0,1)$, then we must have $x=y$. 


\section{Remark 2.1 [2, 22]}

(i) Let us observe that $\delta(r, 0)=0$, and $\delta(r, \varepsilon)$ is an increasing function of $\varepsilon$ for every fixed $r$.

(ii) For $r_{1} \leq r_{2}$ there holds

$$
1-\frac{r_{2}}{r_{1}}\left(1-\delta\left(r_{2}, \varepsilon \frac{r_{1}}{r_{2}}\right)\right) \leq \delta\left(r_{1}, \varepsilon\right) .
$$

(iii) If $(M, d)$ is uniformly convex, then $(M, d)$ is strictly convex.

Lemma 2.1 [2,22] Assume that $(M, d)$ is uniformly convex. Let $\left\{C_{n}\right\} \subset M$ be a sequence of nonempty, nonincreasing, convex, bounded and closed sets. Let $x \in M$ be such that

$$
0<d=\lim _{n \rightarrow \infty} d\left(x, C_{n}\right)<\infty
$$

Let $x_{n} \in C_{n}$ be such that $d\left(x, x_{n}\right) \rightarrow d$. Then $\left\{x_{n}\right\}$ is a Cauchy sequence.

Recall that a hyperbolic metric space $(M, d)$ is said to have the property $(\mathrm{R})$ if any nonincreasing sequence of nonempty, convex, bounded and closed sets has a nonempty intersection [23].

Our next result deals with the existence and the uniqueness of the best approximants of convex, closed and bounded sets in a uniformly convex metric space. This result is of interest by itself as uniform convexity implies the property $(\mathrm{R})$, which reduces to reflexivity in the linear case.

Theorem 2.1 $[2,22]$ Assume that $(M, d)$ is complete and uniformly convex. Let $C \subset M$ be nonempty, convex and closed. Let $x \in M$ be such that $d(x, C)<\infty$. Then there exists a unique best approximant of $x$ in $C$, i.e., there exists a unique $x_{0} \in C$ such that

$$
d\left(x, x_{0}\right)=d(x, C)
$$

The following result gives the analogue of the well-known theorem that states any uniformly convex Banach space is reflexive (see Theorem 2.1 in [16]).

Theorem $2.2[2,22]$ If $(M, d)$ is complete and uniformly convex, then $(M, d)$ has the prop$\operatorname{erty}(\mathrm{R})$.

Note that any hyperbolic metric space $M$ which satisfies the property (R) is complete.

Example 2.1 Let $(X, d)$ be a metric space. A geodesic from $x$ to $y$ in $X$ is a mapping $c$ from a closed interval $[0, l] \subset \mathbb{R}$ to $X$ such that $c(0)=x, c(l)=y$, and $d\left(c(t), c\left(t^{\prime}\right)\right)=\left|t-t^{\prime}\right|$ for all $t, t^{\prime} \in[0, l]$. In particular, $c$ is an isometry and $d(x, y)=l$. The image $\alpha$ of $c$ is called a geodesic (or metric) segment joining $x$ and $y$. The space $(X, d)$ is said to be a geodesic space if every two points of $X$ are joined by a geodesic and $X$ is said to be uniquely geodesic if there is exactly one geodesic joining $x$ and $y$ for each $x, y \in X$, which will be denoted by $[x, y]$ and called the segment joining $x$ to $y$.

A geodesic triangle $\Delta\left(x_{1}, x_{2}, x_{3}\right)$ in a geodesic metric space $(X, d)$ consists of three points $x_{1}, x_{2}, x_{3}$ in $X$ (the vertices of $\Delta$ ) and a geodesic segment between each pair of vertices (the 
edges of $\Delta)$. A comparison triangle for geodesic triangle $\Delta\left(x_{1}, x_{2}, x_{3}\right)$ in $(X, d)$ is a triangle $\bar{\Delta}\left(x_{1}, x_{2}, x_{3}\right):=\Delta\left(\bar{x}_{1}, \bar{x}_{2}, \bar{x}_{3}\right)$ in $\mathbb{R}^{2}$ such that $d_{\mathbb{R}^{2}}\left(\bar{x}_{i}, \bar{x}_{j}\right)=d\left(x_{i}, x_{j}\right)$ for $i, j \in\{1,2,3\}$. Such a triangle always exists (see [24]).

A geodesic metric space is said to be a $\mathrm{CAT}(0)$ space if all geodesic triangles of appropriate size satisfy the following $\mathrm{CAT}(0)$ comparison axiom:

Let $\Delta$ be a geodesic triangle in $X$ and let $\bar{\Delta} \subset \mathbb{R}^{2}$ be a comparison triangle for $\Delta$. Then $\Delta$ is said to satisfy the $\mathrm{CAT}(0)$ inequality if for all $x, y \in \Delta$ and all comparison points $\bar{x}, \bar{y} \in \bar{\Delta}$,

$$
d(x, y) \leq d(\bar{x}, \bar{y})
$$

Complete CAT(0) spaces are often called Hadamard spaces (see [18]). If $x, y_{1}, y_{2}$ are points of a CAT $(0)$ space and $y_{0}$ is the midpoint of the segment $\left[y_{1}, y_{2}\right]$, which will be denoted by $\frac{y_{1} \oplus y_{2}}{2}$, then the CAT $(0)$ inequality implies

$$
d^{2}\left(x, \frac{y_{1} \oplus y_{2}}{2}\right) \leq \frac{1}{2} d^{2}\left(x, y_{1}\right)+\frac{1}{2} d^{2}\left(x, y_{2}\right)-\frac{1}{4} d^{2}\left(y_{1}, y_{2}\right) .
$$

This inequality is the $(\mathrm{CN})$ inequality of Bruhat and Tits [25]. As for the Hilbert space, the $(\mathrm{CN})$ inequality implies that $\mathrm{CAT}(0)$ spaces are uniformly convex with

$$
\delta(r, \varepsilon)=1-\sqrt{1-\frac{\varepsilon^{2}}{4}}
$$

One may also find the modulus of uniform convexity via similar triangles.

\section{Common fixed points of nonexpansive semigroups}

Recall the definition of a nonexpansive mapping defined in metric spaces.

Definition 3.1 Let $(M, d)$ be a metric space and $C \subset M$ be a nonempty subset. A mapping $T: C \rightarrow C$ is said to be nonexpansive if

$$
d(T(x), T(y)) \leq d(x, y)
$$

for any $x, y \in C$. A point $x \in C$ is called a fixed point of $T$ if $T(x)=x$. The set of fixed points of $T$ will be denoted by $\operatorname{Fix}(T)$.

This definition is now extended to a one-parameter family of mappings.

Definition 3.2 Let $(M, d)$ be a metric space and $C \subset M$ be a nonempty subset. A oneparameter family $\mathcal{F}=\left\{T_{t} ; t \geq 0\right\}$ of mappings from $C$ into itself is said to be a nonexpansive semigroup on $C$ if $\mathcal{F}$ satisfies the following conditions:

(i) $T_{0}(x)=x$ for $x \in C$;

(ii) $T_{t+s}(x)=T_{t}\left(T_{s}(x)\right)$ for $x \in C$ and $t, s \in[0, \infty)$;

(iii) for each $t \geq 0, T_{t}$ is a nonexpansive mapping.

Define the set of all common fixed points of $\mathcal{F}$ as

$$
\operatorname{Fix}(\mathcal{F})=\bigcap_{t \geq 0} \operatorname{Fix}\left(T_{t}\right) .
$$


First let us extend Bruck's result [6] to metric spaces.

Lemma 3.1 [6] Let $(M, d)$ be a hyperbolic metric space. Assume that $(M, d)$ is strictly convex. Let $C$ be a subset of $M$. Let $S$ and $T$ be nonexpansive mappings from $C$ into $M$ with a common fixed point. Then, for each $\lambda \in(0,1)$, the mapping $U: C \rightarrow M$ defined by $U(x)=\lambda S(x) \oplus(1-\lambda) T(x)$ for $x \in C$ is nonexpansive and $\operatorname{Fix}(U)=\operatorname{Fix}(S) \cap \operatorname{Fix}(T)$ holds.

Proof Clearly we have $\operatorname{Fix}(S) \cap \operatorname{Fix}(T) \subset \operatorname{Fix}(U)$. Let us now prove that $\operatorname{Fix}(U) \subset \operatorname{Fix}(S) \cap$ $\operatorname{Fix}(T)$. Let $x \in \operatorname{Fix}(U)$, i.e., $U(x)=x$. Since $S$ and $T$ have a common fixed point, let $a \in$ $\operatorname{Fix}(S) \cap \operatorname{Fix}(T)$ be fixed. We have

$$
d(a, x)=d(a, \lambda S(x) \oplus(1-\lambda) T(x)) \leq \lambda d(a, S(x))+(1-\lambda) d(a, T(x))
$$

because of the hyperbolicity of $M$. Since $a$ is a common fixed point of $S$ and $T$ which are nonexpansive, we get

$$
d(a, x)=d(a, \lambda S(x) \oplus(1-\lambda) T(x)) \leq \lambda d(a, x)+(1-\lambda) d(a, x)=d(a, x) .
$$

Therefore we must have

$$
d(a, S(x))=d(a, T(x))=d(a, x)
$$

since $0<\lambda<1$. The strict convexity of $M$ will then imply $S(x)=T(x)$. Clearly from $x=$ $\lambda S(x) \oplus(1-\lambda) T(x)$ we conclude that $S(x)=T(x)=x$, i.e., $x \in \operatorname{Fix}(S) \cap \operatorname{Fix}(T)$. The proof of Lemma 3.1 is complete.

The next result concerns continuous semigroups.

Definition 3.3 Let $(M, d)$ be a metric space and $C \subset M$ be nonempty and closed. A oneparameter family $\mathcal{F}=\left\{T_{t} ; t \geq 0\right\}$ of mappings from $C$ into $M$ is said to be continuous on $C$ if for any $x \in C$, the mapping $t \rightarrow T_{t}(x)$ is continuous, i.e., for any $t_{0} \geq 0$, we have

$$
\lim _{t \rightarrow t_{0}} d\left(T_{t}(x), T_{t_{0}}(x)\right)=0
$$

for any $x \in C$.

The following result is easy to prove.

Proposition 3.1 Let $(M, d)$ be a metric space and $C \subset M$ be nonempty and closed. Let $\mathcal{F}=$ $\left\{T_{t} ; t \geq 0\right\}$ be a one-parameter semigroup of mappings from $C$ into $M$ which is continuous on $C$. Let $A$ be a dense subset of $[0,+\infty)$. Then we have

$$
\operatorname{Fix}(\mathcal{F})=\bigcap_{a \in A} \operatorname{Fix}\left(T_{a}\right)
$$

Recall the following lemma which can be found in any introductory course on real analysis. 
Lemma 3.2 [26] Let $G$ be a nonempty additive subgroup of $\mathbb{R}$. Then $G$ is either dense in $\mathbb{R}$ or there exists $a>0$ such that $G=a \cdot \mathbb{Z}=\{a n, n \in \mathbb{Z}\}$. Therefore if $\alpha$ and $\beta$ are two real numbers such that $\frac{\alpha}{\beta}$ is irrational, then the set

$$
G(\alpha, \beta)=\{\alpha n+\beta m ; n, m \in \mathbb{Z}\}
$$

is dense in $\mathbb{R}$. In particular, the set $G(\alpha, \beta) \cap[0,+\infty)$ is dense in $[0,+\infty)$.

The following result will be useful to prove our main result of this section.

Proposition 3.2 Let $(M, d)$ be a metric space and $C \subset M$ be nonempty and closed. Let $\mathcal{F}=\left\{T_{t} ; t \geq 0\right\}$ be a one-parameter semigroup of mappings from $C$ into $M$. Let $\alpha$ and $\beta$ be any two positive real numbers. Then we have

$$
\bigcap_{a \in G_{+}(\alpha, \beta)} \operatorname{Fix}\left(T_{a}\right)=\operatorname{Fix}\left(T_{\alpha}\right) \cap \operatorname{Fix}\left(T_{\beta}\right)
$$

where $G_{+}(\alpha, \beta)=\{\alpha n+\beta m ; n, m \in \mathbb{Z}\} \cap[0,+\infty)$.

Proof Clearly we have $\bigcap_{a \in G_{+}(\alpha, \beta)} \operatorname{Fix}\left(T_{a}\right) \subset \operatorname{Fix}\left(T_{\alpha}\right) \cap \operatorname{Fix}\left(T_{\beta}\right)$ since $\alpha, \beta \in G_{+}(\alpha, \beta)$. Conversely, let $x \in \operatorname{Fix}\left(T_{\alpha}\right) \cap \operatorname{Fix}\left(T_{\beta}\right)$. Let $a \in G_{+}(\alpha, \beta)$. Then there exist $n, m \in \mathbb{Z}$ such that $a=n \alpha+m \beta$. Assume first that both $n$ and $m$ are positive. Then

$$
T_{a}(x)=T_{n \alpha+m \beta}(x)=T_{n \alpha}\left(T_{m \beta}(x)\right)=T_{\alpha}^{n}\left(T_{\beta}^{m}(x)\right)=x,
$$

where we used the property $T_{0}$ is the identity map. Otherwise assume $a=n \alpha-\bar{m} \beta$, where both $n$ and $\bar{m}$ are positive. Hence $a+\bar{m} \beta=n \alpha$. So

$$
T_{a}(x)=T_{a}\left(T_{\beta}^{\bar{m}}(x)\right)=T_{a+\bar{m} \beta}(x)=T_{n \alpha}(x)=T_{\alpha}^{n}(x)=x .
$$

Hence $x \in \operatorname{Fix}\left(T_{a}\right)$ for any $a \in G_{+}(\alpha, \beta)$. This completes the proof of Proposition 3.2.

If we combine Propositions 3.1 and 3.2, we get the following result.

Theorem 3.1 [27] Let $(M, d)$ be a metric space and $C \subset M$ be nonempty and closed. Let $\mathcal{F}=\left\{T_{t} ; t \geq 0\right\}$ be a one-parameter semigroup of mappings from $C$ into $M$ which is continuous on C. Let $\alpha$ and $\beta$ be two positive real numbers such that $\frac{\alpha}{\beta}$ is irrational, then we have

$$
\operatorname{Fix}(\mathcal{F})=\operatorname{Fix}\left(T_{\alpha}\right) \cap \operatorname{Fix}\left(T_{\beta}\right) .
$$

In particular, we have

$$
\operatorname{Fix}(\mathcal{F})=\operatorname{Fix}\left(T_{1}\right) \cap \operatorname{Fix}\left(T_{\sqrt{2}}\right)=\operatorname{Fix}\left(T_{1}\right) \cap \operatorname{Fix}\left(T_{\pi}\right) .
$$

If the metric space $(M, d)$ is hyperbolic strictly convex, Lemma 3.1 allows us to get the following result. 
Theorem 3.2 Let $(M, d)$ be a hyperbolic metric space. Assume that $(M, d)$ is strictly convex and $C \subset M$ is nonempty and closed. Let $\mathcal{F}=\left\{T_{t} ; t \geq 0\right\}$ be a one-parameter semigroup of nonexpansive mappings from $C$ into $M$ which is continuous on $C$. Let $\alpha$ and $\beta$ be two positive real numbers such that $\frac{\alpha}{\beta}$ is irrational, then we have

$$
\operatorname{Fix}(\mathcal{F})=\operatorname{Fix}\left(\lambda T_{\alpha}+(1-\lambda) T_{\beta}\right)
$$

for any $\lambda \in(0,1)$.

\section{Approximation of common fixed points of semigroups}

In this section we use the previous results to investigate the behavior of Mann iterates generated by two mappings. These iterations will allow us to approximate common fixed points of a continuous semigroup.

Definition 4.1 Let $T: C \rightarrow C$ be a nonexpansive mapping and $\sigma \in(0,1)$. The Mann iteration process generated by the mapping $T$ and the constant $\sigma$, denoted by $M(T, \sigma)$, is defined by the following iteration formula:

$$
x_{n+1}=\sigma T x_{n} \oplus(1-\sigma) x_{n},
$$

where $x_{1}$ is chosen arbitrarily in $C$.

The following technical lemmas will be useful throughout.

Lemma 4.1 $[2,22]$ Let $(M, d)$ be a uniformly convex hyperbolic metric space. Assume that there exists $R \in[0,+\infty)$ such that

$$
\limsup _{n \rightarrow \infty} d\left(x_{n}, a\right) \leq R, \quad \limsup _{n \rightarrow \infty} d\left(y_{n}, a\right) \leq R, \quad \text { and } \quad \lim _{n \rightarrow \infty} d\left(a, \sigma x_{n} \oplus(1-\sigma) y_{n}\right)=R
$$

for some $\sigma \in(0,1)$. Then $\lim _{n \rightarrow \infty} d\left(x_{n}, y_{n}\right)=0$.

Lemma 4.2 Let $(M, d)$ be a uniformly convex hyperbolic metric space. Let $C$ be a nonempty, bounded, closed and convex subset of $M$. Let $T: C \rightarrow C$ be nonexpansive, and let $\sigma \in(0,1)$ and $x_{n}$ be given by (4.1). Assume that $\omega$ is a fixed point of $T$. Then the limit of $\left\{d\left(x_{n}, \omega\right)\right\}$ exists.

Proof We have

$$
\begin{aligned}
d\left(x_{n+1}, \omega\right) & =d\left(\sigma T x_{n} \oplus(1-\sigma) x_{n}, \omega\right) \\
& \leq \sigma d\left(T x_{n}, \omega\right)+(1-\sigma) d\left(x_{n}, \omega\right) \\
& =\sigma d\left(T x_{n}, T \omega\right)+(1-\sigma) d\left(x_{n}, \omega\right) \\
& \leq \sigma d\left(x_{n}, \omega\right)+(1-\sigma) d\left(x_{n}, \omega\right) \\
& =d\left(x_{n}, \omega\right)
\end{aligned}
$$

for any $n \geq 1$. Hence the sequence $\left\{d\left(x_{n}, \omega\right)\right\}$ is decreasing, which implies that it is convergent. 
Let us recall the definition of the asymptotic radius of a sequence.

Definition 4.2 Let $(M, d)$ be a metric space and $C$ be a nonempty subset of $M$. Let $\left\{x_{n}\right\}$ be a bounded sequence in $M$. Define $r\left(\cdot,\left\{x_{n}\right\}\right): C \rightarrow[0, \infty)$ by

$$
r\left(x,\left\{x_{n}\right\}\right)=\limsup _{n \rightarrow \infty} d\left(x, x_{n}\right)
$$

The asymptotic radius $\rho_{C}$ of $\left\{x_{n}\right\}$ with respect to $C$ is given by

$$
\rho_{C}=\inf \left\{r\left(x,\left\{x_{n}\right\}\right): x \in C\right\}
$$

$\rho$ will denote the asymptotic radius of $\left\{x_{n}\right\}$ with respect to $M$. A point $\xi \in C$ is said to be an asymptotic center of $\left\{x_{n}\right\}$ with respect to $C$ if $r\left(\xi,\left\{x_{n}\right\}\right)=r\left(C,\left\{x_{n}\right\}\right)=\min \left\{r\left(x,\left\{x_{n}\right\}\right): x \in C\right\}$.

The set of all asymptotic centers of $\left\{x_{n}\right\}$ with respect to $C$ will be denoted by $A\left(C,\left\{x_{n}\right\}\right)$. When $C=M$, we use the notation $A\left(\left\{x_{n}\right\}\right)$ instead of $A\left(M,\left\{x_{n}\right\}\right)$. In general, the set $A\left(C,\left\{x_{n}\right\}\right)$ of asymptotic centers of a bounded sequence $\left\{x_{n}\right\}$ may be empty or contain more than one point. Note that the asymptotic radius is also known in the literature as a type function. For more on this we refer to [22].

Over the years many people were successful in defining the analogue of linear properties in metric spaces. The weak-topology is still hard to define in the context of metric spaces. An approach to weak-convergence was offered by Kuczumow [28] and Lim [8] which they called $\Delta$-convergence. Their approach was very successful in the case of $\operatorname{CAT}(0)$ spaces.

Definition 4.3 Let $(M, d)$ be a metric space. A bounded sequence $\left\{x_{n}\right\}$ in $M$ is said to $\Delta$ converge to $x \in M$ if and only if $x$ is the unique asymptotic center of every subsequence $\left\{u_{n}\right\}$ of $\left\{x_{n}\right\}$. We write $x_{n} \stackrel{\Delta}{\rightarrow} x$ whenever $\left\{x_{n}\right\} \Delta$-converges to $x$.

In this section, we study the iteration scheme (4.1) for nonexpansive mappings. In particular, we investigate the $\Delta$-convergence in uniformly convex hyperbolic spaces. Note that similar conclusions proved in Banach spaces require the Fréchet differentiability of the norm.

In the sequel, the following results will be needed.

Lemma 4.3 $[22,29]$ Let $(M, d)$ be a hyperbolic metric space. Assume that $M$ is uniformly convex. Let $C$ be a nonempty, closed and convex subset of $M$. Then every bounded sequence $\left\{x_{n}\right\} \in M$ has a unique asymptotic center with respect to $C$.

Lemma 4.4 [22, 29] Let $(M, d)$ be a hyperbolic metric space. Assume that $M$ is uniformly convex. Let $C$ be a nonempty, closed and convex subset of $M$. Let $\left\{x_{n}\right\}$ be a bounded sequence in $C$ such that $A\left(\left\{x_{n}\right\}\right)=\{y\}$ and $r\left(\left\{x_{n}\right\}\right)=\rho$. If $\left\{y_{m}\right\}$ is a sequence in $C$ such that $\lim _{m \rightarrow \infty} r\left(y_{m},\left\{x_{n}\right\}\right)=\rho$, then $\lim _{m \rightarrow \infty} y_{m}=y$.

The following result is similar to the demi-closed principle discovered by Göhde in uniformly convex Banach spaces [30]. 
Lemma 4.5 [29] Let $C$ be a nonempty, closed and convex subset of a complete uniformly convex hyperbolic space $(M, d)$. Let $T: C \rightarrow C$ be a nonexpansive mapping. Let $\left\{x_{n}\right\} \in C$ be an approximate fixed point sequence of $T$, i.e., $\lim _{n \rightarrow \infty} d\left(x_{n}, T x_{n}\right)=0$. If $x \in C$ is the asymptotic center of $\left\{x_{n}\right\}$ with respect to $C$, then $x$ is a fixed point of $T$. In particular, if $\left\{x_{n}\right\} \in C$ is an approximate fixed point sequence of $T$ such that $x_{n} \stackrel{\Delta}{\rightarrow} x$, then $x$ is a fixed point of $T$.

The following theorem is necessary to discuss the behavior of the iterates defined by (4.1).

Theorem 4.1 [29] Let $(M, d)$ be a hyperbolic metric space. Assume that $M$ is uniformly convex. Let $C$ be a nonempty, closed and convex subset of $M$. Let $T: C \rightarrow C$ be a nonexpansive mapping with a nonempty fixed points set. Let $\sigma \in(0,1), x_{1} \in C$ and generate $\left\{x_{n}\right\}$ by (4.1). Then we have

$$
\lim _{n \rightarrow \infty} d\left(T x_{n}, x_{n}\right)=0
$$

i.e., $\left\{x_{n}\right\}$ is an approximate fixed point sequence for $T$.

Proof Let $\omega$ be a fixed point of $T$. From Lemma 4.2, there exists $r \in \mathbb{R}$ such that

$$
\lim _{n \rightarrow \infty} d\left(x_{n}, \omega\right)=r .
$$

Without loss of generality, we may assume $r>0$. Note that

$$
\limsup _{n \rightarrow \infty} d\left(T x_{n}, \omega\right)=\limsup _{n \rightarrow \infty} d\left(T x_{n}, T \omega\right) \leq \limsup _{n \rightarrow \infty} d\left(x_{n}, \omega\right)=r
$$

and

$$
\begin{aligned}
r & =\limsup _{n \rightarrow \infty} d\left(x_{n}, \omega\right)=\limsup _{n \rightarrow \infty} d\left(\sigma T x_{n} \oplus(1-\sigma) x_{n}, \omega\right) \\
& \leq \limsup _{n \rightarrow \infty} \sigma d\left(T x_{n}, \omega\right)+(1-\sigma) d\left(x_{n}, \omega\right) \\
& \leq \limsup _{n \rightarrow \infty} \sigma d\left(x_{n}, \omega\right)+(1-\sigma) d\left(x_{n}, \omega\right) \\
& =\limsup _{n \rightarrow \infty} d\left(x_{n}, \omega\right)=r .
\end{aligned}
$$

Hence

$$
\limsup _{n \rightarrow \infty} d\left(\sigma T x_{n} \oplus(1-\sigma) x_{n}, \omega\right)=r .
$$

Using Lemma 4.1, we conclude

$$
\limsup _{n \rightarrow \infty} d\left(T x_{n}, x_{n}\right)=0 .
$$

Note that the assumption that $T$ has a fixed point may be relaxed if we assume $C$ is bounded (for more see [22]). Next we discuss the $\Delta$-convergence of the Mann iterates defined by (4.1). 
Theorem 4.2 Let $(M, d)$ be a complete hyperbolic metric space. Assume that $M$ is uniformly convex. Let $C$ be a nonempty, closed and convex subset of $M$. Let $T: C \rightarrow C$ be a nonexpansive mapping with a nonempty fixed points set. Let $\sigma \in(0,1), x_{1} \in C$ and generate $\left\{x_{n}\right\}$ by (4.1). Then $\left\{x_{n}\right\} \Delta$-converges to $x$ which is a fixed point of $T$, i.e., $x \in \operatorname{Fix}(T)$.

Proof In [22] it is shown that $\operatorname{Fix}(T)$ is convex and closed. Theorem 4.1 implies that $\left\{x_{n}\right\}$ is an approximate fixed point sequence of $T$, i.e., $\lim _{n \rightarrow \infty} d\left(T x_{n}, x_{n}\right)=0$. Let $x$ be the unique asymptotic center of $\left\{x_{n}\right\}$. Then Lemma 4.5 implies that $x \in \operatorname{Fix}(T)$. Next we show that $\left\{x_{n}\right\}$ $\Delta$-converges to $x$. Let $\left\{x_{n_{i}}\right\}$ be a subsequence of $\left\{x_{n}\right\}$. Let $z$ be the unique asymptotic center of $\left\{x_{n_{i}}\right\}$. Again since $\left\{x_{n_{i}}\right\}$ is an approximate fixed point sequence of $T$, we get $z \in \operatorname{Fix}(T)$. Hence

$$
\limsup _{n_{i} \rightarrow \infty} d\left(x_{n_{i}}, z\right) \leq \limsup _{n_{i} \rightarrow \infty} d\left(x_{n_{i}}, x\right) .
$$

Since $x, z \in \operatorname{Fix}(T)$, we get

$$
\limsup _{n_{i} \rightarrow \infty} d\left(x_{n_{i}}, z\right)=\lim _{n \rightarrow \infty} d\left(x_{n}, z\right), \quad \text { and } \quad \limsup _{n_{i} \rightarrow \infty} d\left(x_{n_{i}}, x\right)=\lim _{n \rightarrow \infty} d\left(x_{n}, x\right) .
$$

Since $x$ is the unique asymptotic center of $\left\{x_{n}\right\}$, we get $x=z$. This proves that $\left\{x_{n}\right\} \Delta$ converges to $x$.

If we combine Theorem 3.2 and Theorem 4.2, we obtain the following result.

Theorem 4.3 Let $(M, d)$ be a complete hyperbolic metric space. Assume that $M$ is uniformly convex. Let $C$ be a nonempty, closed and convex subset of $M$. Let $\mathcal{F}=\left\{T_{t} ; t \geq 0\right\}$ be a one-parameter semigroup of nonexpansive mappings from $C$ into $C$. Assume that $\mathcal{F}$ is continuous and has a nonempty common fixed points set. Let $\alpha$ and $\beta$ be two positive real numbers such that $\frac{\alpha}{\beta}$ is irrational. Fix $\lambda, \mu \in(0,1)$ such that $\lambda+\mu<1$. Let $x_{1} \in C$ and define a sequence $\left\{x_{n}\right\}$ in $C$ by

$$
x_{n+1}=(\mu+\lambda)\left(\mu T_{\alpha}\left(x_{n}\right) \oplus \lambda T_{\beta}\left(x_{n}\right)\right) \oplus(1-\mu-\lambda) x_{n}
$$

for any $n \geq 1$. Then $\left\{x_{n}\right\} \Delta$-converges to a common fixed point of the semigroup $\mathcal{F}$.

Proof Set

$$
S=\frac{\mu}{\mu+\lambda} T_{\alpha} \oplus \frac{\lambda}{\mu+\lambda} T_{\beta}
$$

Observe that $S: C \rightarrow C$ is nonexpansive. Clearly we have $\operatorname{Fix}(\mathcal{F}) \subset \operatorname{Fix}(S)$. Let $x_{1} \in C$ and $\left\{x_{n}\right\}$ be the sequence generated by (4.4). Then

$$
x_{n+1}=\sigma S\left(x_{n}\right)+(1-\sigma) x_{n},
$$

where $\sigma=\mu+\lambda \in(0,1)$. Clearly $\left\{x_{n}\right\}$ is the same sequence generated by (4.1) for the map $S$. Theorem 4.2 implies that $\left\{x_{n}\right\} \Delta$-converges to some $x \in \operatorname{Fix}(S)$. From Theorem 3.2, we 
know that

$\operatorname{Fix}(\mathcal{F})=\operatorname{Fix}(S)$.

Hence $x \in \operatorname{Fix}(\mathcal{F})$, which completes the proof of Theorem 4.3.

Using the techniques developed in [29], one may show that the conclusion of Theorem 4.3 is still valid if we consider the modified Mann iteration

$$
x_{n+1}=\alpha_{n} T_{\alpha}\left(\beta_{n} T_{\beta}\left(x_{n}\right) \oplus\left(1-\beta_{n}\right) x_{n}\right) \oplus\left(1-\alpha_{n}\right) x_{n},
$$

where $\alpha_{n}, \beta_{n} \in(0,1)$.

\section{Competing interests}

The authors declare that they have no competing interests.

\section{Authors' contributions}

All authors contributed equally to the writing of this paper. All authors read and approved the final manuscript.

\section{Author details}

'Department of Mathematics, Faculty of Science For Girls, King Abdulaziz University, P.O. Box 80203, Jeddah, 21589, Saudi Arabia. ${ }^{2}$ Department of Mathematical Sciences, The University of Texas at El Paso, El Paso, TX 79968, USA. ${ }^{3}$ Department of Mathematics \& Statistics, King Fahd University of Petroleum and Minerals, Dhahran, 31261, Saudi Arabia.

\section{Acknowledgements}

This work was funded by the Deanship of Scientific Research (DSR), King Abdulaziz University, Jeddah. The authors, therefore, acknowledge technical and financial support of KAU.

Received: 1 August 2014 Accepted: 14 January 2015 Published online: 30 April 2015

References

1. Khamsi, MA, Kozlowski, WK: On asymptotic pointwise contractions in modular function spaces. Nonlinear Anal. 73 , 2957-2967 (2010)

2. Khamsi, MA, Kozlowski, WK: On asymptotic pointwise nonexpansive mappings in modular function spaces. J. Math. Anal. Appl. 380, 697-708 (2011)

3. Belluce, LP, Kirk, WA: Fixed-point theorems for families of contraction mappings. Pac. J. Math. 18, $213-217$ (1966)

4. Belluce, LP, Kirk, WA: Nonexpansive mappings and fixed-points in Banach spaces. III. J. Math. 11, 474-479 (1967)

5. Browder, FE: Nonexpansive nonlinear operators in a Banach space. Proc. Natl. Acad. Sci. USA 54, 1041-1044 (1965)

6. Bruck, RE: Properties of fixed-point sets of nonexpansive mappings in Banach spaces. Trans. Am. Math. Soc. 179, 251-262 (1973)

7. DeMarr, RE: Common fixed-points for commuting contraction mappings. Pac. J. Math. 13, 1139-1141 (1963)

8. Lim, TC: A fixed point theorem for families of nonexpansive mappings. Pac. J. Math. 53, 487-493 (1974)

9. Tan, K-K, Xu, H-K: An ergodic theorem for nonlinear semigroups of Lipschitzian mappings in Banach spaces. Nonlinear Anal. 19(9), 805-813 (1992)

10. Kirk, WA, Xu, HK: Asymptotic pointwise contractions. Nonlinear Anal. 69, 4706-4712 (2008)

11. Hussain, N, Khamsi, MA: On asymptotic pointwise contractions in metric spaces. Nonlinear Anal. 71(10), 4423-4429 (2009)

12. Khamsi, MA: Nonlinear semigroups in modular function spaces. Math. Jpn. 379, 1-9 (1992)

13. Menger, K: Untersuchungen über allgemeine Metrik. Math. Ann. 100, 75-163 (1928)

14. Reich, S, Shafrir, I: Nonexpansive iterations in hyperbolic spaces. Nonlinear Anal. 15, 537-558 (1990)

15. Busemann, H: Spaces with non-positive curvature. Acta Math. 80, 259-310 (1948)

16. Goebel, K, Reich, S: Uniform Convexity, Hyperbolic Geometry, and Nonexpansive Mappings. Series of Monographs and Textbooks in Pure and Applied Mathematics, vol. 83. Dekker, New York (1984)

17. Kirk, WA: Fixed Point Theory for Nonexpansive Mappings, I and II. Lecture Notes in Mathematics, vol. 886, pp. 485-505 Springer, Berlin (1981)

18. Kirk, WA: A fixed point theorem in CAT(0) spaces and R-trees. Fixed Point Theory Appl. 2004(4), 309-316 (2004)

19. Leustean, L: A quadratic rate of asymptotic regularity for CAT(0)-spaces. J. Math. Anal. Appl. 325, 386-399 (2007)

20. Clarkson, JA: Uniformly convex spaces. Trans. Am. Math. Soc. 40(3), 396-414 (1936)

21. Goebel, K, Sekowski, T, Stachura, A: Uniform convexity of the hyperbolic metric and fixed points of holomorphic mappings in the Hilbert ball. Nonlinear Anal. 4, 1011-1021 (1980)

22. Khamsi, MA, Khan, AR: Inequalities in metric spaces with applications. Nonlinear Anal. TMA 74, 4036-4045 (2011)

23. Khamsi, MA: On metric spaces with uniform normal structure. Proc. Am. Math. Soc. 106, 723-726 (1989)

24. Bridson, M, Haefliger, A: Metric Spaces of Non-Positive Curvature. Springer, Berlin (1999)

25. Bruhat, F, Tits, J: Groupes réductifs sur un corps local. I. Données radicielles valuées. Publ. Math. Inst. Hautes Études Sci. 41, 5-251 (1972) 
26. Stewart, I, Tall, D: Algebraic Number Theory and Fermat's Last Theorem, 3rd edn. AK Peters, Wellesley (2001)

27. Suzuki, T: The set of common fixed points of a one-parameter continuous semigroup of mappings is $F(T(1)) \cap F(T(\sqrt{2}))$. Proc. Am. Math. Soc. 134(3), 673-681 (2006)

28. Kuczumow, T: An almost convergence and its applications. Ann. Univ. Mariae Curie-Skłodowska, Sect. A 32, 79-88 (1978)

29. Fukhar-ud-din, H, Khamsi, MA: Approximating common fixed points in hyperbolic spaces. Fixed Point Theory Appl. 2014, $113(2014)$

30. Göhde, D: Zum prinzip der kontraktiven abbildung. Math. Nachr. 30, 251-258 (1965)

Submit your manuscript to a SpringerOpen ${ }^{\odot}$ journal and benefit from:

- Convenient online submission

- Rigorous peer review

- Immediate publication on acceptance

- Open access: articles freely available online

- High visibility within the field

- Retaining the copyright to your article 